\title{
Efficacy and Safety of Bivalirudin versus Heparin Plus Tirofiban in Elderly Patients with Acute Myocardial Infarction Undergoing Primary Percutaneous Coronary Intervention
}

\author{
Ke Zhu, Wen Lu, Yi-jie Huang, Lin-guang Wang, Qiang Wu, Chun-guang Feng and Qiang Fu* \\ Department of Cardiology, Xuzhou Hospital Affiliated East South University School of Medicine, Xuzhou Cardiovascular Disease Institute, PR China
}

\begin{abstract}
Objective: The aim of this study was to investigate the antithrombotic effect and safety of Bivalirudin compared with heparin plus Tirofiban in elderly patients with acute myocardial infarction undergoing primary Percutaneous Coronary Intervention (PCl).

Methods: One hundred and twenty elderly patients were randomly assigned to receive two different antithrombotic therapies (Group A: Bivalirudin alone or Group B: Heparin plus Tirofiban) in a 1:1 ratio according to the treatment sequence and a table of random numbers. The clinical information, routine examination results, infarct-related sites of the enrolled patients were recorded, and the Thrombolysis in Myocardial Infarction (TIMI) flow grade and other
\end{abstract} safety indexes after $\mathrm{PCl}$ were analyzed.

Results: No statistically significant differences existed in ST-segment depression at $2 \mathrm{~h}$ after intervention, post$\mathrm{PCI}$ TIMI flow grade and reduction of N-terminal pro-B type natriuretic peptide (NT-proBNP) serum concentrations in patients on day 7 versus day 1 after $\mathrm{PCl}$ between the two groups $(P>0.05)$. However, the adverse clinical events of bleeding showed significant differences in the two groups $(P<0.05)$.

Conclusion: Bivalirudin exerts confirmed anticoagulant effect and indicates lower risk of bleeding compared with heparin plus Tirofiban in elderly patients with acute myocardial infarction undergoing primary PCl.

Keywords: Bivalirudin; Heparin; Tirofiban; Percutaneous coronary intervention; Efficacy

\section{Introduction}

Percutaneous Coronary Intervention (PCI), a therapy that can improve myocardial blood supply using cardiac catheterization technique to dilate stenotic or occlusive coronary arteries, performs relative small trauma and low risk [1,2]. Antithrombotic drugs are needed for the patients before and after PCI. Heparin, as the first choice of commonly used drugs in the past, has displayed rapid and obvious effect. Nevertheless, heparin has several well-established limitations, including unpredictable anticoagulant effect and high bleeding risk [3]. Bivalirudin, a new anticoagulant drug, can bind with thrombin directly, specifically and reversibly, and shows better efficacy, safety and tolerance compared with heparin [4]. Moreover, bivalirudin has been widely used in clinical as an anticoagulant therapy $[5,6]$ including Percutaneous Coronary Artery forming (PTCA), Unstable Angina Pectoris (UAP), Acute Coronary Syndrome (ACS) and Myocardial Infarction (MI), peripheral artery interventional therapy, major heart surgery, heart-lung transplantation and so on and high rank recommendations by correlation guide and so on ACC, ESC $[7,8]$. The current study aims to evaluate the antithrombotic effect and safety of Bivalirudin compared with Heparin plus Tirofiban in 120 elderly patients with acute ST-Segment Elevation Myocardial Infarction (STEMI) undergoing primary PCI.

\section{Methods}

\section{Study population}

One hundred and twenty consecutive elderly patients (93 men and 27 women) aged from 60 to 82 years (mean \pm SD, $70.98 \pm 4.06$ years), The time of onset to PCI was less than 12 hours, who were hospitalized in Department of Cardiology, Xuzhou Hospital Affiliated East South
University School of Medicine from February 2013 to August 2015 because of STEMI undergoing primary PCI, were enrolled. Patients were randomly assigned to receive two different antithrombotic therapies (Group A: bivalirudin alone or Group B: heparin plus tirofiban) in a 1:1 ratio according to the treatment sequence and a table of random numbers. The enrolled patients were eligible for STEMI diagnostic criteria of American College of Cardiology (ACC) and European Society of Cardiology (ESC). Exclusion criteria included previous or current serious heart, liver, renal or other critical organ failures, neurological or psychiatric diseases, major surgery or trauma history, systemic infection, patients in the menstrual period, pregnancy, lactation and other special populations. All patients provided written informed consent before randomization.

\section{Treatment}

Patients in the two groups were treated with the same routine support therapy. All patients were pretreated before intervention with aspirin $(300 \mathrm{mg}$ ) and clopidogrel $(600 \mathrm{mg})$. For Group A, bivalirudin was given as an intravenous bolus of $0.75 \mathrm{mg} / \mathrm{kg}$ before intervention,

*Corresponding author: Qiang Fu, Department of Cardiology, Xuzhou Hospital Affiliated East South University School of Medicine, Xuzhou Cardiovascular Disease Institute, 199 Jiefang Road, Xuzhou 221009, PR China, Tel: +86 18952170277; E-mail: fuqiang@medmail.com.cn

Received January 21, 2016; Accepted February 01, 2016; Published February 08, 2016

Citation: Zhu K, Lu W, Huang Y, Wang L, Wu Q, et al. (2016) Efficacy and Safety of Bivalirudin versus Heparin Plus Tirofiban in Elderly Patients with Acute Myocardial Infarction Undergoing Primary Percutaneous Coronary Intervention. Cardiovasc Pharm Open Access 5: 172. doi:10.4172/2329-6607.1000172

Copyright: (c) 2016 Zhu K, et al. This is an open-access article distributed under the terms of the Creative Commons Attribution License, which permits unrestricted use, distribution, and reproduction in any medium, provided the original author and source are credited. 
Citation: Zhu K, Lu W, Huang Y, Wang L, Wu Q, et al. (2016) Efficacy and Safety of Bivalirudin versus Heparin Plus Tirofiban in Elderly Patients with Acute Myocardial Infarction Undergoing Primary Percutaneous Coronary Intervention. Cardiovasc Pharm Open Access 5: 172. doi:10.4172/2329-6607.1000172

Page 2 of 4

followed by infusion of $1.75 \mathrm{mg} / \mathrm{kg} / \mathrm{h}$ during the PCI procedure and for 4 hours afterwards. For Group B, heparin $100 \mathrm{U} / \mathrm{kg}$ (maximum dose was $10000 \mathrm{U}$ ) and tirofiban $10 \mu \mathrm{g} / \mathrm{kg}$ boluses were given before intervention, followed by a $0.75 \mu \mathrm{g} / \mathrm{kg} / \mathrm{min}$ tirofiban infusion during the PCI procedure and for 36 hours afterwards. After the PCI, additional low-molecular-weight heparin $(5000 \mathrm{U}, 2$ times/day for 7 days) was administered as a subcutaneous injection, aspirin $100 \mathrm{mg} /$ day and clopidogrel bisulfate $75 \mathrm{mg} /$ day were administered orally. Other cardiovascular medications, such as statins, beta blockers or nitrates, were given in accordance with the patient's condition. All interventions were performed with the same equipment's and operators.

\section{End points and definitions}

General information, medical history, routine physical examination, including blood lipids, blood glucose, blood pressure, routine blood test, hemoglobin of all patients were recorded. All patients were observed until seven days after PCI. Taking Thrombolysis in Myocardial Infarction (TIMI) flow grades as main indexes of the clinical evaluation. The sites of myocardial infarction and TIMI flow grades in the infarct-related artery were obtained. And the rates of ST-segment depression were measured at $2 \mathrm{~h}$ after PCI. The electrochemical luminescence immunity analysis method was used to detect the reduction of $\mathrm{N}$-terminal pro- $\mathrm{B}$ type natriuretic peptide (NT-proBNP) serum concentrations in patients on day 7 versus day 1 after PCI to evaluate statistically significant differences between the two groups in the myocardial perfusion level and the improvement of cardiac function. Bleeding is the main safety indexes and any bleeding as defined by the Bleeding Academic Research Consortium (BARC) definition was observed.

\section{Statistical analysis}

All calculations were performed by SPSS 17.0. Numerical values were expressed as mean $\pm \mathrm{SD}$ and analyzed with $\mathrm{t}$-test between the Group. Median with interquartile range (IQR) Q50 (Q25Q75)) were reported when the data are not normally distributed. Logtransformation was applied before using the t-test to compare the two groups. Categorical variables were expressed as percentage and compared using the $\chi^{2}$ test. Fisher's exact tests were used to compare two categorical variables when expected numbers are small. A value of $P<0.05$ was considered statistically significant.

\section{Results}

\section{Clinical characteristics}

The baseline characteristics of this study population are indicated in Table 1 . No statistically significant differences were observed in age, gender, medical history, infarct-related artery and clinical examinations between the two randomization groups.

\section{Treatment and procedural characteristics}

Bivalirudin significantly reduced the rates of hemoptysis and Bleeding Academic Research Consortium (BARC) type 2 bleeding in patients with acute myocardial infarction undergoing primary $\mathrm{PCI}$, compared with heparin plus tirofiban $(P<0.05$, Table 2$)$.

\section{Discussion}

Heparin plus Tirofiban has been commonly used as the antithrombotic therapy during PCI in clinical. Heparin can accelerate

\begin{tabular}{|c|c|c|c|c|}
\hline Characteristic & Bivalirudin $(n=60)$ & Heparin Plus Tirofiban $(n=60)$ & $t$ or $x^{2}$ & $P$ \\
\hline Gender, male/female & $48 / 12$ & $45 / 15$ & 0.05 & $>0.05$ \\
\hline Age (yr) & $71.34 \pm 4.03$ & $70.73 \pm 3.58$ & 0.40 & $>0.05$ \\
\hline Diabetes mellitus & 18 & 19 & 0.04 & $>0.05$ \\
\hline Smoking history & 21 & 22 & 0.04 & $>0.05$ \\
\hline $\mathrm{T}$ (onset to pci) & $5.52 \pm 0.90$ & $5.63 \pm 0.97$ & 0.22 & $>0.05$ \\
\hline \multicolumn{5}{|l|}{ Clinical examination } \\
\hline Hemoglobin $(g / L)$ & $120 \pm 12$ & $119 \pm 14$ & 0.42 & $>0.05$ \\
\hline Dyslipidemia & 46 & 47 & 0.05 & $>0.05$ \\
\hline $\mathrm{TC}(\mathrm{mmol} / \mathrm{L})$ & $6.48 \pm 1.23$ & $6.42 \pm 1.32$ & 0.26 & $>0.05$ \\
\hline $\mathrm{TG}(\mathrm{mmol} / \mathrm{L})$ & $1.96 \pm 0.98$ & $1.97 \pm 1.02$ & 0.05 & $>0.05$ \\
\hline LDL (mmol/L) & $3.63 \pm 1.03$ & $3.62 \pm 1.23$ & 0.05 & $>0.05$ \\
\hline $\mathrm{HDL}(\mathrm{mmol} / \mathrm{L})$ & $1.02 \pm 0.42$ & $1.03 \pm 0.53$ & 0.11 & $>0.05$ \\
\hline Hypertension & 43 & 44 & 0.04 & $>0.05$ \\
\hline \multicolumn{5}{|l|}{ Infarct-related site } \\
\hline Anterior MI & 31 & 28 & 0.30 & $>0.05$ \\
\hline Un-anterior Ml & 29 & 32 & 0.30 & $>0.05$ \\
\hline \multicolumn{5}{|l|}{ Infarct-related artery } \\
\hline LAD & 23 & 21 & 0.14 & $>0.05$ \\
\hline LCX & 18 & 23 & 0.93 & $>0.05$ \\
\hline RCA & 19 & 16 & 0.36 & $>0.05$ \\
\hline
\end{tabular}

TC: Total Cholesterol; TG: Triglyceride; LDL: Low-Density Lipoprotein; HDL: High-Density Lipoprotein; LAD: Left Anterior Descending; LCX: Left Circumflex Coronary Artery; RCA: Right Coronary Artery.

Table 1: Baseline characteristics. 
Citation: Zhu K, Lu W, Huang Y, Wang L, Wu Q, et al. (2016) Efficacy and Safety of Bivalirudin versus Heparin Plus Tirofiban in Elderly Patients with Acute Myocardial Infarction Undergoing Primary Percutaneous Coronary Intervention. Cardiovasc Pharm Open Access 5: 172. doi:10.4172/2329-6607.1000172

Page 3 of 4

\begin{tabular}{|c|c|c|c|c|}
\hline Characteristic & Bivalirudin $(n=60)$ & $\begin{array}{l}\text { Heparin Plus Tirofiban } \\
(n=60)\end{array}$ & $t$ or $x^{2}$ & $P$ \\
\hline \multicolumn{5}{|c|}{ ST-segment depression at $2 \mathrm{~h}$ after $\mathrm{PCl}, \mathrm{NO} .(\%)$} \\
\hline$<30 \%$ & $8(13.33)$ & $3(5.00)$ & 2.50 & $>0.05$ \\
\hline $30 \%-70 \%$ & $7(11.67)$ & $4(6.67)$ & 0.90 & $>0.05$ \\
\hline$>70 \%$ & $45(75.00)$ & $53(88.33)$ & 3.56 & $>0.05$ \\
\hline \multicolumn{5}{|l|}{ TIMI flow, No. (\%) } \\
\hline TIMI flow 0 & $2(3.33)$ & $4(6.67)$ & 0.18 & $>0.05$ \\
\hline TIMI flow 1 & $5(8.33)$ & $6(10.00)$ & 0.10 & $>0.05$ \\
\hline TIMI flow 2 & $8(13.33)$ & $7(11.67)$ & 0.08 & $>0.05$ \\
\hline TIMI flow 3 & $45(75.00)$ & $43(71.67)$ & 0.17 & $>0.05$ \\
\hline NT-proBNP reduction (pg/ml) & $723(304,1506)$ & $719(289,1497)$ & 0.12 & $>0.05$ \\
\hline \multicolumn{5}{|l|}{ Bleeding definition, NO. (\%) } \\
\hline Intracerebral hemorrhage & $0(0.00)$ & $1(1.67)$ & - & $>0.05$ \\
\hline Gastrointestinal bleeding & $1(1.67)$ & $6(10.00)$ & 2.43 & $>0.05$ \\
\hline Hemoptysis & $0(0.00)$ & $9(15.00)$ & - & $<0.05$ \\
\hline BARC 2, NO. (\%) & $2(3.33)$ & $13(21.67)$ & 9.22 & $<0.05$ \\
\hline Blood transfusion, NO. (\%) & $0(0.00)$ & $1(1.67)$ & - & $>0.05$ \\
\hline
\end{tabular}

Table 2: Treatment and procedural characteristics.

the activation of antithrombin and inhibit the activity of thrombin, but it can be caused bleeding when used excessively. Tirofiban attribute to restore blood flow of coronary artery infarction but can increase the risk of bleeding [9]. Bivalirudin, a new direct thrombin inhibitor, is an oligopeptide analogue of hirudin. Bivalirudin has performed more sufficient anticoagulant effect by binding with thrombin directly, specifically and reversibly. Therefore, bivalirudin is especially suitable for the patients who need high intensity anticoagulation therapy in a short term or the ones with high bleeding risk. In our study, bivalirudin or heparin plus tirofiban was administrated as antithrombotic therapy for the 120 elderly patients with acute myocardial infarction undergoing primary PCI, indicating no statistically significant differences between the two groups in the myocardial perfusion level and the improvement of cardiac function $(P>0.05)$, including ST-segment depression at $2 \mathrm{~h}$ after intervention, post-PCI TIMI flow grade, the reduction of NT-proBNP serum concentrations of patients on day 7 versus day 1 after PCI. The results indicate that bivalirudin can act as an effective antithrombotic therapy for emergency PCI, which is the same as the past researches $[10,11]$. Another large-scale clinical study of bivalirudin has found that bivalirudin performs similar anticoagulant effect as heparin, yet it shows the most important advantage for its little bleeding adverse effect as the antithrombotic therapy for PCI, peripheral vascular interventional therapy, heart-lung transplantation and renal dysfunction [12]. The current study also shown that the rate of bleeding in Group A was reduced considerably by bivalirudin compared with that in Group B received heparin plus tirofiban $(P<0.05)$, which was in concordance with the reported researches. There appeared 1 case of gastrointestinal hemorrhage and no cases of intracerebral hemorrhage, hemoptysis in Group A, yet 1 case of intracerebral hemorrhage, 6 cases of gastrointestinal hemorrhage and 9 cases of hemoptysis in Group B. Furthermore, the rate of BARC type 2 bleeding in Group A was lower than that in Group B $(P<0.05)$. These results indicate that bivalirudin can significantly reduce the risk of bleeding compared with heparin plus tirofiban.
In conclusion, this study showed that bivalirudin was effective and safe with lower risks of bleeding compared with heparin plus tirofiban as an anticoagulant therapy for the patients with acute myocardial infarction undergoing emergency PCI. These findings indicated bivalirudin can be used as a routine antithrombotic drug for PCI in clinical.

\section{References}

1. Diletti R, Van Mieghem NM, Zijlstra F (2015) Primary PCl with or without Thrombectomy. The New England journal of medicine 373: 682-683.

2. Shao Y, Chen MT, Zhang S (2013) Effect of early beta blocker therapy in patients with acute ST-segment elevation myocardial infarction undergoing percutaneous coronary intervention on long term prognosis. Chin J Cardiol 41: 659-661.

3. Abtahian F, Waldo S, Jang IK (2015) Comparison of heparin and bivalirudin in patients undergoing percutaneous coronary intervention without use of glycoprotein Ilb/llla inhibitors. Catheterization and cardiovascular interventions: official journal of the Society for Cardiac Angiography \& Interventions 86: 390396.

4. Stone GW, Witzenbichler B, Guagliumi G, Peruga JZ, Brodie BR, et al. (2011) Heparin plus a glycoprotein Ilb/IIla inhibitor versus bivalirudin monotherapy and paclitaxel-eluting stents versus bare-metal stents in acute myocardial infarction (HORIZONS-AMI): final 3-year results from a multicentre, randomised controlled trial [J]. The Lancet 377: 2193-2204.

5. Bisehof D, Dalbert S, Zollinger A, Ganter MT, Hofer CK (2010) Thrombelastography in the surgical patient[J]. Minerva Anestesiol 76: 131-137.

6. Deshpande NV, Pratiti R, Admane P, Mukherjee D, Mardikar HM (2012) Safety and efficacy of bivalirudin with glycoprotein Ilb/Illa for high-risk percutaneous coronary intervention. Indian Heart J 64: 444-448.

7. O'Gara PT, Kushner FG, Ascheim DD, Casey DE Jr, Chung MK, et al. (2013) 2013 ACCF/AHA guideline for the management of ST-elevation myocardia infarction:a report of the American College of Cardiology Foundation/American Heart Association Task Force on Practice Guidelines [J]. J Am Coll Cardiol 61: 78-140.

8. Roffi M, Patrono C, Collet JP, Marco Valgimigli, Felicita Andreotti, et al. (2015) 2015 ESC Guidelines for the Management of Acute Coronary Syndromes in 
Citation: Zhu K, Lu W, Huang Y, Wang L, Wu Q, et al. (2016) Efficacy and Safety of Bivalirudin versus Heparin Plus Tirofiban in Elderly Patients with Acute Myocardial Infarction Undergoing Primary Percutaneous Coronary Intervention. Cardiovasc Pharm Open Access 5: 172. doi:10.4172/2329-6607.1000172

Patients Presenting Without Persistent ST-segment Elevation. [J]. Revista espanola de cardiologia (English ed.) 68: 1125.

9. Woehrle J, Parise H, Mehran R, Dudek D, Kornowski R, et al. (2013) Impact of bivalirudin and paclitaxel-eluting stents on outcomes in patients undergoing primary percutaneous coronary intervention of the left anterior descending artery. Am J Cardiol 112: 753-60.

10. Sethi A, Bahekar A, Doshi H, Bhuriya R, Bedi U, et al. (2011) Tirofiban use with clopidogrel and aspirin decreases adverse cardiovascular events after percutaneous coronary intervention for ST-elevation myocardial infarction: a meta-analysis of randomized trials. Canadian Journal of Cardiology 27: 548554.

11. Xiang DC, Gu XL, Pan CM (2011) Evaluation of anticoagulant efficacy and safety for domestic bivalirudin during percutaneous coronary intervention. Chinese Circulation Journal 26: 331-334.

12. Berger PB, Janzen MC, Manoukian SV (2013) CRT-33 Peri-procedural CK-MB levels in percutaneous coronary intervention with high-dose bolus tirofiban vs. abciximab plus either unfractionated heparin or bivalirudin: an analysis from Tenacity. JACC: Cardiovascular Interventions 6: S11. 